\title{
Maintenance of bovine oocytes in prophase of meiosis I by high $[\mathrm{cAMP}]_{\mathrm{i}}$
}

\author{
H. Aktas ${ }^{1 *}$, M. B. Wheeler ${ }^{2}$, C. F. Rosenkrans, $\mathrm{Jr}^{1 \dagger}$, N. L. First ${ }^{1}$ and \\ M. L. Leibfried-Rutledge ${ }^{1+}$ \\ 'Department of Meat and Animal Science, University of Wisconsin, Madison, WI 53706 USA; and \\ ${ }^{2}$ Department of Animal Sciences, University of Illinois, Urbana, IL 61801 USA
}

\begin{abstract}
The effects of high intracellular cAMP concentrations ([cAMP $]_{i}$ ) on germinal vesicle maintenance of bovine cumulus-oocyte complexes were investigated, using 8-bromo- $3^{\prime}, 5^{\prime}$ cAMP (8-Br-cAMP) or an invasive adenylate cyclase from Bordetella pertussis to increase the $[c A M P]_{\mathrm{i}}$. The effects of interactions of these agents with macromolecular supplements in culture medium (fetal calf serum, FCS; polyvinylpyrrolidone, PVP; BSA), and different methods of processing complexes before culture, on subsequent germinal vesicle maintenance by invasive adenylate cyclase were studied. While 8-Br-cAMP was unable to maintain germinal vesicle arrest in the majority of oocytes for $20 \mathrm{~h}(36 \%$ with FCS, $24 \%$ with BSA, $18 \%$ with PVP), it maintained germinal vesicle arrest in a high proportion of cumulusenclosed oocytes when BSA or PVP was used $(37 \%$ with FCS, $52 \%$ with BSA, $53 \%$ with $P V P)$. The difference in frequency of germinal vesicle maintenance between macromolecular supplements was not related to $[\mathrm{cAMP}]_{\mathrm{i}}$ when assayed after culture for $2 \mathrm{~h}$ with invasive adenylate cyclase. Complexes processed in whole follicular fluid were not maintained in meiotic arrest $(26 \%)$ when cultured with invasive adenylate cyclase and PVP. Complexes processed in follicular fluid with 3-isobutyl I-methylxanthine (IBMX) plus invasive adenylate cyclase were arrested at the germinal vesicle stage at high frequencies $(65 \%)$, while those processed in IBMX or IBMX plus 8-Br-cAMP-supplemented follicular fluid had intermediate ( $43 \%$ and $49 \%$, respectively) frequencies of intact germinal vesicles. Oocyte complexes processed in follicular fluid supplemented with IBMX and invasive adenylate cyclase formed morulae and blastocysts $(27.2 \%)$, as did oocytes processed in follicular fluid alone $(26 \%)$. Phosphoprotein profiles showed that control oocytes and 8-Br-cAMP-treated oocytes share a profile that is different from that of oocytes treated with invasive adenylate cyclase. These results show that increased $[\mathrm{cAMP}]_{i}$ reversibly maintains bovine oocytes in meiotic arrest for an extended period without the occurrence of the post-translational protein modifications observed during meiotic resumption or transient arrest.
\end{abstract}

\section{Introduction}

Oocytes of most mammals begin meiosis during fetal development and arrest at prophase I (germinal vesicle, GV, stage). Oocytes overcome this arrest after either hormonal induction in situ or spontaneously after removal from antral follicles. The mechanism by which oocytes are kept in meiotic arrest in antral follicles has been the subject of many investigations. Cho et al. (1974) first implicated intracellular cAMP ([cAMP $]_{i}$ ) in maintaining meiotic arrest in mammals. In both mice (Schultz et al., 1983; Vivarelli et al., 1983) and rats (Aberdam et al., 1987),

\footnotetext{
*Present address: Dana-Farber Cancer Institute, Harvard Medical School, Boston, MA 02115, USA.

${ }^{\dagger}$ Present address: Department of Animal Sciences. University of Arkansas, Fayetteville, AR 72701, USA.

${ }^{\ddagger}$ Reprint requests.

Received 28 December 1994
}

agents that maintain high intracellular concentrations of cAMP prevent spontaneous oocyte maturation. In mice (Schultz et al, 1983; Vivarelli et al., 1983) and rats (Aberdam et al., 1987), spontaneous maturation of oocytes is correlated with decreased [cAMP $]_{i}$ in both cumulus-oocyte complexes and oocytes. In amphibians (Maller and Krebs, 1977), in which hormonal induction of meiotic maturation is required, agents that maintain high $[\mathrm{cAMP}]_{i}$ aiso interfere with the induction of meiotic maturation.

Studies of domestic species with long oestrous cycles, compared with laboratory species with short cycles, have failed to produce such clear-cut results and increases in $[\mathrm{cAMP}]_{\mathrm{i}}$ elicit only a transient delay in meiotic resumption, as evaluated by germinal vesicle breakdown. Whereas testosterone and a cAMP analogue maintain meiotic arrest cooperatively in pig oocytes, cAMP alone gives only a small decrease in maturation frequency (Rice and McGaughey, 1981). Bovine oocytes respond 
to agents with only a transient maintenance of meiotic arrest (Homa, 1988; Sirard and First, 1988), with the exception of $\mathrm{NaF}$ (Sirard, 1990) or pharmacological doses of 3-isobutyl 1-methyl xanthine (IBMX; Homa, 1988). The effects of NaF are irreversible, while the reversibility of IBMX has not been reported.

Agents such as cholera toxin and forskolin that directly stimulate adenylate cyclase by affecting regulatory G-proteins do cause an increase in [cAMP $]_{i}$ in pig (Racowsky, 1985a) and sheep (Crosby et al., 1985) oocytes, but either do not maintain (Crosby et al., 1985) or only transiently maintain (Racowsky, 1985a; Sirard, 1990) meiotic arrest. Adenylate cyclase is found in cattle oocytes (Kuyt et al., 1988), but it is unclear whether it contributes significantly to $[\mathrm{cAMP}]_{\mathrm{i}}$ in domestic species or whether cumulus cells are the main mediators for cAMP concentrations in oocytes, as has been proposed for laboratory species (Racowsky, 1984; Bornslaeger and Schultz, 1985). It is possible that, although meiotic arrest is dependent on high $[\mathrm{CAMP}]_{\mathrm{i}}$, resumption of meiosis $\mathrm{I}$ in oocyte complexes is not dependent on decreasing $[\mathrm{cAMP}]_{i}$ in the face of gonadotrophin stimulation. As has been suggested in mice (Downs et al., 1988) and cows (Aktas et al., 1991a; Aktas, 1994), a positive signal originating from the follicle cells surrounding the oocyte may cause meiotic resumption despite unaltered [cAMP] $]_{\mathrm{i}}$.

A series of studies to determine the effects of agents that increase $[\mathrm{cAMP}]_{i}$ on the maintenance of meiotic arrest in vitro was initiated to clarify the role of $[\mathrm{CAMP}]_{\mathrm{i}}$ in mediating meiotic arrest in one species with long cycles, i.e. cattle. We report here that an invasive adenylate cyclase (iAC) increases $[\mathrm{CAMP}]_{i}$ both in bovine cumulus cells and the oocyte, and keeps the oocyte arrested at the germinal vesicle stage for an extended period. The arrest is reversible and success of arrest is affected by both macromolecular supplement in the medium and procedures for recovering the oocyte-cumulus complexes from the ovary. Parts of this study have been reported as an abstract (Aktas et al., 1990).

\section{Materials and Methods}

\section{Oocyte recovery and culture}

Bovine ovaries were obtained at an abattoir (Pecks, Milwaukee, WI) and transported to the laboratory in thermos bottles containing saline $(0.9 \% \mathrm{w} / \mathrm{v} \mathrm{NaCl})$ at $35-36^{\circ} \mathrm{C}(4-6 \mathrm{~h}$ for collection and transport). Temperature on arrival at the laboratory averaged $31^{\circ} \mathrm{C}$. Contents were aspirated from antral follicles $2-4 \mathrm{~mm}$ in diameter. When $5 \mathrm{ml}$ of pooled follicular fluid (FF) from the experimental groups had been collected, it was supplemented with one of the agents that increase intracellular $[\mathrm{cAMP}]_{\mathrm{i}}$, depending on the individual experiments. Follicular fluid from control groups was not supplemented unless stated otherwise. Complete aspiration, washing and selection of the oocytes took an average of 2-3 h until all treatment groups were filled for a replicate. Oocytes with an intact, compact cumulus investment were selected and washed twice in $\mathrm{CO}_{2}$-equilibrated TC-199 with Earle's salts (Gibco, Grand Island, NY), using a stereomicroscope and were then transferred to culture (Leibfried and First, 1979).

In Expts 1 and 2, the interaction between agents increasing oocyte $[\mathrm{CAMP}]_{\mathrm{i}}$ and different macromolecular supplements in the culture medium was investigated. All reagents were purchased from Sigma (St Louis, MO), unless otherwise stated. Either 10 mmol 8-bromo-3'5' $\mathrm{cAMP} \mathrm{l}^{-1}$ (8-Br-cAMP), shown by Homa (1988) to be the most effective analogue and the most effective concentration for bovine oocytes, or $20 \mathrm{U}$ iAC $\mathrm{ml}^{-1}$ from Bordetella pertussis (kindly provided by E. Hewlett, University of Virginia Medical School; activity of the enzyme that maintained $50 \%$ bovine denuded oocytes in meiotic arrest was considered $10 \mathrm{U}$, arbitrarily) were used to increase $[\mathrm{CAMP}]_{\mathrm{i}}$. Heat-treated fetal calf serum (FCS; $\left.10 \% \mathrm{v} / \mathrm{v}, \mathrm{Gibco}\right)$, BSA ( $3 \mathrm{mg} \mathrm{ml}^{-1}$, Fraction V) or polyvinylpyrrolidone (PVP; $40 \mathrm{kDa}$ molecular mass, $3 \mathrm{mg} \mathrm{ml}^{-1}$ ) were used as macromolecular supplements. Oocytes were cultured in TC-199 medium (with Earle's salts) supplemented with pyruvate $\left(0.2 \mathrm{mmol} \mathrm{l}^{-1}\right)$, gentamicin $\left(25 \mu \mathrm{g} \mathrm{ml}{ }^{-1}\right)$ and meiotic inhibitors, as required for a particular experiment. Treatment groups were supplemented with $10 \mathrm{mmol} 8$-Br-cAMP I- ${ }^{-1}$ for Expt 1 , and $20 \mathrm{U}$ invasive adenylate cyclase $\mathrm{ml}^{-1}$ for Expt 2. Oocytes were cultured in 96-well culture dishes, with 25 complexes in $125 \mu \mathrm{l}$ medium in $5 \% \mathrm{CO}_{2}$ in air with high humidity at $39^{\circ} \mathrm{C}$ for $20 \mathrm{~h}$. Follicular fluid aspirated from antral follicles was supplemented with $0.5 \mathrm{mmol}$ IBMX $1^{-1}+0.5 \mathrm{mmol} 8-\mathrm{Br}$ cAMP $1^{-1}$ for Expt 1 and $0.5 \mathrm{mmol}$ IBMX $\mathrm{l}^{-1}+2 \mathrm{U}$ invasive adenylate cyclase $\mathrm{ml}^{-1}$ for Expt 2 . In these and all experiments, all treatments were represented in each replicate.

Experiment 3 was designed to compare different preculture treatments that might prevent commitment before culture (i.e., during aspiration, collection and washing). Follicular fluid was supplemented with either IBMX $\left(0.5 \mathrm{mmol} \mathrm{l}^{-1}\right), \mathrm{IBMX}+8-\mathrm{Br}-$ CAMP $\left(0.5 \mathrm{mmol} \mathrm{l}^{-1}\right), \mathrm{IBMX}+$ invasive adenylate cyclase ( $2 \mathrm{U}$ $\mathrm{ml}^{-1}$ ) or there was no supplement. Again, each treatment was represented in every replicate. Oocytes collected from each preculture treatment were randomly assigned for subsequent culture in TC-199 with PVP, with or without invasive adenylate cyclase, for $20 \mathrm{~h}$. Intracellular cAMP in whole complexes and cumulus-free oocytes was measured to determine whether differences in maintenance of germinal vesicle arrest among different treatments in Expts 2 and 3 were related to differences in initial CAMP concentrations when invasive adenylate cyclase was present. In Expt 2, cAMP was measured after oocytes had been in culture for $2 \mathrm{~h}$. In Expt 3, [cAMP $]_{i}$ was measured after processing, at the time when oocytes would normally be put in to culture.

Experiment 4 was designed to study the reversibility of inhibition by invasive adenylate cyclase. Cumulus-oocyte complexes derived from FF treated with IBMX $(0.5 \mathrm{mmol}$ $\left.\mathrm{I}^{-1}\right)+$ invasive adenylate cyclase $\left(2 \mathrm{U} \mathrm{ml}^{-1}\right)$ and subsequently cultured in culture medium supplemented with PVP and invasive adenylate cyclase $\left(20 \mathrm{U} \mathrm{ml}^{-1}\right)$ for $20 \mathrm{~h}$ were washed twice in pre-equilibrated TC-199, transferred to TC-199 + PVP without invasive adenylate cyclase and incubated for an additional $20 \mathrm{~h}$. A group of cumulus-oocyte complexes recovered from non-supplemented FF and cultured without invasive adenylate cyclase served as the control.

\section{Preparation of oocytes for fertilization and embryo culture}

In Expt 5, oocytes (ten in a drop) recovered from FF without supplements or FF supplemented with IBMX + invasive 
adenylate cyclase were subsequently cultured in TC-199 with LH $\left(5 \mu \mathrm{g} \mathrm{ml}^{-1}\right), \mathrm{FSH}\left(0.5 \mu \mathrm{g} \mathrm{ml}^{-1}\right)$ (both gonadotrophins were ovine derivatives provided by $\mathrm{NIH}$ ), oestradiol ( $\mathrm{I} \mu \mathrm{g}$ $\left.\mathrm{ml}^{-1}\right)$, gentamicin $\left(25 \mu \mathrm{g} \mathrm{ml}^{-1}\right)$ and pyruvate $\left(0.2 \mathrm{mmol} \mathrm{l}^{-1}\right)$ in $50 \mu \mathrm{l}$ droplets under paraffin oil in $5 \% \mathrm{CO}_{2}$ with high humidity at $39^{\circ} \mathrm{C}$ (Lenz et al., 1983) for $22 \mathrm{~h}$ (Sirard et al., 1988). Frozen-thawed semen (American Breeders Service, DeForest, WI) was used for fertilization using a swim-up procedure (Parrish et al., 1986). Fertilization medium was a modified Tyrode's solution supplemented with fatty-acid-free BSA $\left(6 \mathrm{mg} \mathrm{ml}^{-1}\right.$ ), pyruvate, gentamicin, hypotaurine, penicillamine, adrenaline and heparin (Leibfried and Bavister, 1982; Parrish et al., 1986). Glucose was eliminated from the formulation. The final concentration of spermatozoa was $10^{6} \mathrm{ml}^{-1}$. Embryos were stripped of cumulus cells and transferred onto oviductal cell monolayers (Eyestone and First, 1989) $48 \mathrm{~h}$ after insemination, cultured for an additional 4 days and then scored for morulae and blastocyst formation.

\section{Radiolabelling and two-dimensional gel electrophoresis of oocyte phosphoproteins}

Experiment 6 compared phosphoprotein profiles of oocytes recovered and cultured with 8-Br-cAMP, or invasive adenylate cyclase, or in control media for a total of $9 \mathrm{~h}$. Cumulus-oocyte complexes from each treatment were cultured in phosphatefree TC-199 medium supplemented with $0.5 \mu \mathrm{Ci}\left[{ }^{32} \mathrm{P}\right]$ orthophosphate $\mathrm{ml}^{-1}$ (Amersham, Arlington Heights, IL) during the last $2 \mathrm{~h} 15 \mathrm{~min}$. At the end of the labelling period, the cumulus cells were removed in phosphate-free TC-199 and washed in phosphate-free TALP-Hepes solution (Bavister et al., 1983). Seventy-five oocytes were pooled in $4 \mu \mathrm{l}$, flash-frozen in liquid nitrogen and stored at $-20^{\circ} \mathrm{C}$ until electrophoresis.

Before electrophoresis, the samples were frozen and thawed several times and $1 \mu \mathrm{RNAse}-\mathrm{DNAse}\left(20 \mathrm{mmol}\right.$ Tris- $\mathrm{HCl}^{-1}$, $10 \mathrm{mmol} \mathrm{MgCl}_{2} \mathrm{l}^{-1}, 60 \mathrm{mmol} \mathrm{NaF} \mathrm{I}^{-1}, 2.2 \mathrm{mmol} \mathrm{PMSF} \mathrm{l}^{-1}$, $0.5 \mathrm{mg}$ RNAse $\mathrm{ml}^{-1}$ and $0.1 \mathrm{mg}$ DNAse $\mathrm{ml}^{-1}$ ) was added. Samples were incubated on ice for $30 \mathrm{~min}$. A lysis buffer $\left(9.5 \mathrm{~mol}\right.$ urea $\mathrm{l}^{-1}, 10 \% \mathrm{w} / \mathrm{v}$ CHAPS, $1.5 \% \mathrm{pH} 5-7,1.5 \%$ pH 6-8 and 1\% pH 3-10 ampholytes; Bio-Rad, Herculus CA) with a trace of fast green was added. The samples were centrifuged at $16000 \mathrm{~g}$ for $10 \mathrm{~min}$, and the supernatant was loaded onto tube gels $(7 \mathrm{~cm}$ in length). The gel mixture was $9.15 \mathrm{~mol}$ urea $\mathrm{l}^{-1}, 10 \%(\mathrm{w} / \mathrm{v})$ CHAPS, $4 \%$ acrylamide and ampholines (1.5\% pH 5-7, 1.5\% pH 6-8, 1\% pH 3-10). The upper chamber buffer was $20 \mathrm{mmol} \mathrm{NaOH} \mathrm{l}^{-1}$ and the lower chamber buffer was $10 \mathrm{mmol} \mathrm{H}_{3} \mathrm{PO}_{4} \mathrm{l}^{-1}$. The gels were initially run for $10 \mathrm{~min}$ at $200 \mathrm{~V}, 15 \mathrm{~min}$ at $300 \mathrm{~V}$ and $15 \mathrm{~min}$ at $400 \mathrm{~V}$ before the samples were loaded. Loaded gels were run at $750 \mathrm{~V}$ for $3.5 \mathrm{~h}$. The first dimension gels were extruded, incubated in solubilization buffer and loaded onto $8-15 \%(\mathrm{w} / \mathrm{v})$ SDS slab gels with molecular weight markers (Laemmli, 1970). Silver-stained gels were dried between two pieces of cellophane paper under a vacuum. Preflashed X-ray film (X-AR-OMAT) was exposed for 3 days with intensifying screens, developed and the pixel intensities normalized using Collage (Photodyne, Hartland, WI) software. The data were analysed by completely randomized design to eliminate errors associated with replicates.

\section{Measurements of $c A M P$}

Oocytes were either stripped of cumulus cells by repeatedly aspirating complexes through a glass pipette $(200 \mu \mathrm{m}$ i.d.), or left intact and washed three times at $4^{\circ} \mathrm{C}$ in the presence of $0.5 \mathrm{mmol}$ IBMX I ${ }^{-1}$ in a modified Tyrode's solution without glucose (TL-Hepes; Bavister et al., 1983). A fourth wash was carried out under the same conditions in the absence of IBMX. Each experiment was performed three times and duplicate samples were obtained for each replicate. Samples of five oocyte-cumulus complexes or 50 denuded oocytes were transferred to $150 \mu \mathrm{l}$ extraction medium (TC-199) in $10 \mu \mathrm{l}$ of the last wash. Similar amounts of the last wash were used as blanks for each group and the cAMP concentration (background noise) in this blank was subtracted from the cAMP concentration measured from corresponding samples. Trichloroacetic acid (10 $\mu \mathrm{l}$ of $100 \% \mathrm{w} / \mathrm{v}, \mathrm{TCA})$ was added to the samples, and they were then vortexed for $30 \mathrm{~s}$ and centrifuged at $16000 \mathrm{~g}$ for $8 \mathrm{~min}$ at $4^{\circ} \mathrm{C}$. Twice the volume of a 4:6 mixture of tri-n-octylamine (Sigma) and 1,1,2trichlorotrifluoroethane (Aldrich, Milwaukee, WI) were added to extract the TCA (Chen et al., 1977; Schoff et al,, 1989). Samples were frozen in liquid nitrogen and stored at $-70^{\circ} \mathrm{C}$ until assayed. A radioimmunoassay kit from Biomedical Technologies, Inc. (Stoughton, MA) was used to measure cAMP; the sensitivity of the assay was $5 \mathrm{fmol}$ and highly specific antibodies raised against succinyl cAMP-tyrosine methyl ester were used. ${ }^{125}$ I] succinyl cAMP-tyrosine methyl ester was used as a tracer.

The assay was validated by adding increasing numbers $(20$, $40,60,80$ ) of oocytes to a constant volume of extraction medium, as well as by adding standard amounts of cAMP to samples and recovering proportional amounts of cAMP. No allowance was made for extraction losses which were presumed to be $5-10 \%$ (Chen et al., 1977).

\section{Data evaluation and statistical analysis}

For evaluation of meiotic stage after culture, oocytes were mounted on slides beneath a coverslip supported by $3: 1$ petroleum jelly:paraffin, fixed and cleared in acid alcohol (3:1 ethanol:acetic acid) and examined at $\times 400$ magnification using Nomarski optics. Oocytes were classified as: germinal vesicle stage, with intact nuclear membranes; intermediate, if after germinal vesicle breakdown to metaphase I; or mature, if at anaphase I to metaphase II.

Percentage data were analysed by one-way analysis of variance (ANOVA) with or without arcsin transformation, and the same results were obtained in each case. Means were compared using the Newman-Keuls method, if ANOVA revealed significant differences between treatments. The percentage of oocytes remaining at the germinal vesicle stage was used as the end point for comparison of treatments with inhibitors. The percentage of oocytes matured was the end point for comparison of control groups and reversal of invasive adenylate cyclase maintained arrest. The percentage of morulae and blastocysts was used as the end point for embryo development. The content of cAMP (fmol) per oocyte or per oocyte-cumulus complex was used as the end point for comparison of CAMP measurements. The cAMP data (obtained 
Table 1. Effect of 8-bromo cAMP (8-Br-cAMP) on germinal vesicle (GV) maintenance in bovine cumulus-oocyte complexes cultured with different macromolecular supplements

\begin{tabular}{lcrcr}
\hline Supplement $^{\mathrm{a}}$ & $\begin{array}{c}\text { 8-Br-cAMP } \\
\left(\mathrm{mmol} \mathrm{l}^{-1}\right)\end{array}$ & $\begin{array}{c}\text { GV } \\
(\%) \pm \mathrm{SEM}^{\mathrm{b}}\end{array}$ & $\begin{array}{c}\text { Intermediate } \\
(\%) \pm \text { SEM }^{\mathrm{b}}\end{array}$ & $\begin{array}{c}\text { Mature } \\
(\%) \pm \mathrm{SEM}^{\mathrm{b}}\end{array}$ \\
\hline FCS & 10 & $36 \pm 7.4$ & $58 \pm 6.9$ & $6 \pm 2.4$ \\
FCS & 0 & $7 \pm 2.1$ & $23 \pm 3.8$ & $70 \pm 3.7$ \\
PVP & 10 & $18 \pm 10.7$ & $66 \pm 10.2$ & $16 \pm 2.4$ \\
PVP & 0 & $6 \pm 3.0$ & $19 \pm 5.3$ & $75 \pm 4.0$ \\
BSA & 10 & $26 \pm 5.1$ & $55 \pm 4.1$ & $19 \pm 4.5$ \\
BSA & 0 & $2 \pm 2.2$ & $28 \pm 2.8$ & $70 \pm 2.8$ \\
\hline
\end{tabular}

FCS: fetal calf serum; PVP: polyvinylpyrrolidone.

aCumulus-oocyte complexes were collected in follicular fluid with 3-isobutyl 1-methylxanthine (IBMX $\left.0.5 \mathrm{mmol} \mathrm{l}^{-1}\right)+8$ bromo-cAMP $\left(0.5 \mathrm{mmol} \mathrm{l} \mathrm{l}^{-1}\right.$ ) added at $5 \mathrm{ml}$ volume. After three washes in control medium, complexes were placed in TC-199 plus indicated additives for $20 \mathrm{~h}$.

${ }^{\mathrm{S}}$ Total number of oocytes within each treatment ranged from 71 to 100 over five replications. No difference between treatments was observed.

in conjunction with Expt 3) were analysed both by including and excluding samples derived from follicular fluid supplemented with IBMX + 8-Br-cAMP. Correlations between cAMP concentration and the frequency of oocytes remaining at the germinal vesicle stage were calculated by simple linear regression after excluding the treatment group derived from FF supplemented with IBMX +8 -Br-cAMP.

\section{Results}

Interaction of macromolecular supplements with 8-Br-cAMP

The results from Expt 1 , in which cumulus-oocyte complexes recovered from FF supplemented with $\mathrm{IBMX}+8-\mathrm{Br}-$ cAMP were cultured for $20 \mathrm{~h}$ with different macromolecular supplements (BSA, PVP, FCS) with or without 8-Br-cAMP $\left(10 \mathrm{mmol} \mathrm{l}^{-1}\right)$, are given in Table 1 . Regardless of macromolecular supplement, only a small proportion of the oocytes remained at the germinal vesicle stage after $20 \mathrm{~h}$ of culture when incubated with 8-Br-cAMP. The frequency of germinal vesicle arrest did not differ statistically between treatments containing 8-Br-cAMP. The majority of the oocytes were at metaphase I, indicating a transient delay in meiotic resumption. No further attempt was made to use lower or higher doses, since this has been done by others (Homa, 1988). Oocytes in control groups reached metaphase II at reasonably high frequencies regardless of the macromolecular supplement present.

\section{Interaction of invasive adenylate cyclase with different protein supplements}

In Expt 2, oocytes were recovered from FF supplemented with IBMX + invasive adenylate cyclase and cultured for $20 \mathrm{~h}$ with different macromolecular supplements (FCS, PVP, BSA), with or without $20 \mathrm{U}$ invasive adenylate cyclase $\mathrm{ml}^{-1}$ (Table 2). Oocytes cultured in medium containing FCS had a lower
Table 2. Effect of adenylate cyclase on germinal vesicle (GV) maintenance in bovine cumulus-oocyte complexes cultured with different macromolecular supplements

\begin{tabular}{lcccr}
\hline Supplement & $\begin{array}{c}\text { iAC } \\
\left(\mathrm{U} \mathrm{ml}^{-1}\right)\end{array}$ & $\begin{array}{c}\text { GV } \\
(\%) \pm \text { SEM }^{\mathrm{b}}\end{array}$ & $\begin{array}{c}\text { Intermediate } \\
(\%) \pm \text { SEM }^{\mathrm{b}}\end{array}$ & $\begin{array}{c}\text { Mature } \\
(\%) \pm \mathrm{sEM}^{\mathrm{b}}\end{array}$ \\
\hline FCS & 20 & $36 \pm 3.8^{\mathrm{c}}$ & $60 \pm 3.7$ & $4 \pm 2.2$ \\
FCS & 0 & $8 \pm 5.4$ & $37 \pm 3.9$ & $55 \pm 4.1$ \\
BSA & 20 & $52 \pm 3.7^{\mathrm{d}}$ & $46 \pm 4.3$ & $2 \pm 1.9$ \\
BSA & 0 & $7 \pm 3.0$ & $32 \pm 4.6$ & $61 \pm 2.2$ \\
PVP & 20 & $53 \pm 4.7^{\mathrm{d}}$ & $43 \pm 5.1$ & $3 \pm 1.8$ \\
PVP & 0 & $16 \pm 3.4$ & $37 \pm 2.7$ & $47 \pm 4.3$ \\
& & & &
\end{tabular}

FCS: fetal calf serum; PVP: polyvinylpyrrolidone; $\mathrm{A} A \mathrm{C}$ : invasive adenylate cyclase.

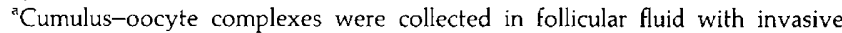
cyclase $\left(2 \mathrm{U} \mathrm{ml}^{-1}\right)$ plus 3-isobutyl 1-methylxanthine (IBMX; $0.5 \mathrm{mmol} \mathrm{l}^{-1}$ ) added at $5 \mathrm{ml}$ volume. After three washes in control medium, complexes were placed in TC-199 medium plus the indicated additives for $20 \mathrm{~h}$.

${ }^{b}$ The total number of oocytes within each treatment ranged from 82 to 120

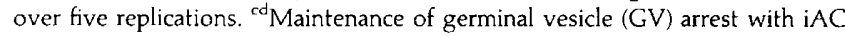
present within treatments with different superscripts is significantly different $(P<0.05)$. The percentage intermediate was not analysed.

Table 3. $[\mathrm{CAMP}]_{i}$ in bovine oocyte complexes and in oocytes cultured with adenylate cyclase and different macromolecular supplements

\begin{tabular}{lcc}
\hline $\begin{array}{l}\text { Macromolecular } \\
\text { supplement }\end{array}$ & $\begin{array}{c}\text { CAMP } \\
(\text { fmol per complex })^{\mathbf{a}} \\
\pm \text { SEM }\end{array}$ & $\begin{array}{c}\text { CAMP } \\
(\text { fmol per oocyte })^{\mathrm{a}} \\
\pm \text { SEM }\end{array}$ \\
\hline FCS & $119.4 \pm 32.7$ & $7.7 \pm 0.7$ \\
PVP & $152.8 \pm 11.4$ & $7.5 \pm 1.6$ \\
BSA & $177.0 \pm 21.8$ & $9.0 \pm 1.7$ \\
\hline
\end{tabular}

FCS: fetal calf serum; PVP: polyvinylpyrrolidone.

${ }^{a}$ Complexes were incubated for $2 \mathrm{~h}$ in invasive adenylate cyclase $\left(20 \mathrm{U} \mathrm{ml}^{-1}\right)$, and then processed for cAMP assay. Some were stripped of cumulus (50 oocytes in a sample), while others were measured as whole complexes (five complexes in a sample). Duplicate samples were obtained for every treatment within each replicate. Values are means for three replications.

frequency $(P<0.05)$ of germinal vesicle maintenance $(36 \%)$ than did those cultured with either PVP $(53 \%)$ or BSA $(52 \%)$. Approximately half of the oocytes cultured with either BSA or PVP in the presence of invasive adenylate cyclase maintained an intact germinal vesicle for $20 \mathrm{~h}$. Most of the remaining oocytes in these three treatments also showed a transient delay in germinal vesicle breakdown, since few were classified as meiotically mature at $20 \mathrm{~h}$. Again, oocytes matured equally well in all three macromolecular supplements.

In conjunction with this experiment, cAMP concentrations in whole complexes and in oocytes alone were measured after the complexes had been cultured in invasive adenylate cyclase $\left(20 \mathrm{U} \mathrm{ml}^{-1}\right)$ for $2 \mathrm{~h}$ with different macromolecular supplements to determine whether differences in frequency of germinal vesicle maintenance could be correlated with differences in cAMP concentrations (Table 3). Neither complexes nor 
Table 4. Effect of preculture collection and wash procedures on germinal vesicle (GV) maintenance in bovine oocyte complexes subsequently cultured with invasive adenylate cyclase (iAC)

\begin{tabular}{lcccr}
\hline $\begin{array}{l}\text { Inhibitor in } \\
\text { follicular fluid }\end{array}$ & $\begin{array}{c}\mathrm{iAC} \\
\left(\mathrm{U} \mathrm{ml}^{-\mathrm{I}}\right)\end{array}$ & $\begin{array}{c}\mathrm{GV} \\
(\%) \pm \mathrm{SEM}\end{array}$ & $\begin{array}{c}\text { Intermediate } \\
(\%) \pm \mathrm{SEM}\end{array}$ & $\begin{array}{c}\text { Mature } \\
(\%) \pm \mathrm{SEM}\end{array}$ \\
\hline $\mathrm{IBMX}+\mathrm{iAC}$ & 20 & $65 \pm 2.8^{\mathrm{b}}$ & $31 \pm 4.7$ & $4 \pm 1.5$ \\
& 0 & $10 \pm 3.1$ & $33 \pm 5.2$ & $57 \pm 5.0$ \\
IBMX+ & 20 & $49 \pm 4.9^{\mathrm{c}}$ & $43 \pm 5.1$ & $9 \pm 2.7$ \\
$8-\mathrm{Br}-\mathrm{CAMP}$ & 0 & $10 \pm 2.9$ & $37 \pm 3.7$ & $53 \pm 4.4$ \\
IBMX & 20 & $43 \pm 4.9^{\mathrm{c}}$ & $52 \pm 5.4$ & $5 \pm 2.1$ \\
& 0 & $11 \pm 5.1$ & $33 \pm 4.6$ & $56 \pm 6.6$ \\
None & 20 & $26 \pm 5.0^{\mathrm{d}}$ & $62 \pm 3.8$ & $13 \pm 3.4$ \\
& 0 & $9 \pm 2.7$ & $24 \pm 4.4$ & $67 \pm 3.5$
\end{tabular}

${ }^{3} 3$-Isobutyl 1-methylxanthine (IBMX) and 8-bromo cAMP (8-Br-cAMP) were used at $0.5 \mathrm{mmol} \mathrm{l}^{-1}, \mathrm{iAC}$ at $2 \mathrm{U} \mathrm{ml}^{-1}$. The total number of oocytes used within a treatment ranged from 83 to 110 over five replications.

${ }^{b}-d V a l u e s$ for treatments having $\mathrm{iAC}$ present with different superscripts are significantly different $(P<0.05)$. The percentage of intermediates was not analysed.

cumulus-free oocytes had statistically different cAMP concentrations, suggesting that the lower frequency of germinal vesicle maintenance, when cultured with FCS, was due to interaction of yet unknown factors in FCS with cAMP. The use of FCS as a supplement in culture medium when studying meiotic arrest was discontinued from this point. In this experiment no control measurements were made of untreated oocytes or complexes; however, such observations were made in a later experiment (Table 5). Comparison of the estimates of cAMP concentrations show that they were about ten times higher in the presence of invasive adenylate cyclase.

\section{Effect of supplementing follicular fluid during collection on subsequent maintenance of meiotic arrest}

Sirard and First (1988) suggested that bovine oocytes commit to undergo meiosis soon after removal from the follicle. Our preliminary studies also suggested that care should be taken to prevent premature meiotic commitment during aspiration, collection and wash procedures. For that reason, it was necessary to develop a practical system to prevent commitment while collecting and washing the bovine material. This was done in Expt 3. Follicular fluid was supplemented with IBMX $\left(0.5 \mathrm{mmol} \mathrm{l}^{-1}\right)$, IBMX plus 8-Br-cAMP $\left(0.5 \mathrm{mmol} \mathrm{l}^{-1}\right)$, IBMX plus invasive adenylate cyclase $\left(2 \mathrm{U} \mathrm{ml}^{-1}\right)$, or for the controls there was no supplement. Oocytes recovered from these four different preculture collection treatments were allocated randomly for culture with or without invasive adenylate cyclase $\left(20 \mathrm{U} \mathrm{ml}^{-1}\right)$ for $20 \mathrm{~h}$ (Table 4). Concentrations of cAMP in complexes or oocytes were measured at the time when they would have been transferred to culture, to determine whether preculture procedures affected $[\mathrm{CAMP}]_{i}$ at the onset of culture (Table 5).

Oocytes from FF supplemented with IBMX + invasive adenylate cyclase had the highest frequency of germinal vesicles $(65 \%)$, whereas arrested oocytes from FF supplemented with IBMX alone $(43 \%)$ or IBMX plus 8 -Br-cAMP $(49 \%)$ were
Table 5. $[\mathrm{CAMP}]_{i}$ after preculture procedures for collecting bovine cumulus-oocyte complexes

\begin{tabular}{lcc}
\hline $\begin{array}{l}\text { Inhibitor added to } \\
\text { follicular fluid }\end{array}$ & $\begin{array}{c}\text { CAMP } \\
\text { (fmol per complex) } \\
\pm \text { SEM }\end{array}$ & $\begin{array}{c}\text { cAMP } \\
\text { (fmol oocyte per) } \\
\pm \text { SEM }\end{array}$ \\
\hline IBMX + iAC & $27.9 \pm 4.5^{\mathrm{a}}$ & $0.97 \pm 0.16^{\mathrm{a}}$ \\
IBMX +8Br-cAMP & $240.4 \pm 94.7^{\mathrm{b}}$ & $13.47 \pm 5.90^{\mathrm{b}}$ \\
IBMX & $15.2 \pm 2.9^{\mathrm{c}}$ & $0.54 \pm 0.05^{\mathrm{c}}$ \\
None & $10.9 \pm 1.5^{\mathrm{c}}$ & $0.50 \pm 0.04^{\mathrm{c}}$ \\
\hline
\end{tabular}

IBMX: 3-isobutyl 1-methylxanthine; $\mathrm{AAC}$ : invasive adenylate cyclase; 8 Br-cAMP: 8-bromo cAMP.

${ }^{a-c}$ Within a column, values for treatments with different superscripts are significantly different $(P<0.05)$. Means are presented for three replications. Fifty denuded oocytes and five complexes were assayed per sample. Duplicate samples of each treatment were taken at every replication.

intermediate (Table 4). The oocytes treated with invasive adenylate cyclase recovered from unsupplemented FF $(26 \%)$ were similar to controls $(P>0.05)$. These data suggest that bovine oocytes begin commitment to undergo meiotic maturation if they are kept in FF for $\geq 2 \mathrm{~h}$. None of the pretreatments affected the frequency of maturation in control groups.

The $[\mathrm{cAMP}]_{\mathrm{i}}$ of both complexes $(28 \mathrm{fmol})$ and oocytes $(0.97 \mathrm{fmol})$ recovered from IBMX $+\mathrm{iAC}$ FF supplement was higher than that of complexes $(10.9 \mathrm{fmol})$ or oocytes $(0.5 \mathrm{fmol})$ derived from unsupplemented FF $(P<0.05$, Table 5$)$ when measured after preculture treatments. The complexes and the oocytes recovered from FF with IBMX added had intermediate [cAMP $_{i}(15.15$ and $0.54 \mathrm{fmol}$ in complexes and oocytes, respectively), which correlates with their degree of retention of the germinal vesicle. Oocytes recovered from $\mathrm{FF}$ supplemented with IBMX plus 8-Br-cAMP had very high contents of [cAMP $]_{i}$, mostly attributable to the CAMP analogue, yet maintenance of meiotic arrest was only comparable with oocytes recovered from FF supplemented with IBMX alone. The role of cAMP in meiotic arrest was assessed by estimating the correlation between the maintenance of the germinal vesicle and the concentration of cAMP after the different pretreatments. The correlation was 0.93 for cumulus-oocyte complexes and 0.78 for denuded oocytes, when oocytes derived from IBMX + 8-Br-cAMP supplemented FF were not considered. This group was excluded from further analysis as representing an outlier.

\section{Reversibility of inhibition}

Cumulus-oocyte complexes recovered from IBMX + invasive adenylate cyclase supplemented FF and cultured in the presence of $\mathrm{iAC}$ for $20 \mathrm{~h}$ were washed twice and cultured in the same medium without invasive adenylate cyclase for an additional $20 \mathrm{~h}$ to test whether the effect of $\mathrm{iAC}$ on oocytes was reversible. The results (Table 6) show that inhibition of germinal vesicle breakdown by invasive adenylate cyclase was reversible. Although not statistically different, the slightly lower frequency for completion of meiotic maturation may be 
Table 6. Reversibility of meiotic arrest in vitro of bovine oocytes maintained by invasive adenylate cyclase (iAC)

\begin{tabular}{lccc}
\hline $\begin{array}{l}\text { Inhibitor } \\
\text { in culture }\end{array}$ & $\begin{array}{c}\text { GV } \\
(\%) \pm \text { SEM }\end{array}$ & $\begin{array}{c}\text { Intermediate } \\
(\%) \pm \text { SEM }\end{array}$ & $\begin{array}{c}\text { Mature } \\
(\%) \pm \text { SEM }\end{array}$ \\
\hline iAC & $58 \pm 4.4^{\mathrm{b}}$ & $41 \pm 3.3$ & $3 \pm 1.4^{\mathrm{b}}$ \\
Control $_{\text {Reversal }}^{\mathrm{a}}$ & $11 \pm 5.1^{\mathrm{c}}$ & $29 \pm 5.8$ & $60 \pm 6.7^{\mathrm{c}}$ \\
\hline
\end{tabular}

GV: germinal vesicle.

${ }^{\mathrm{a}}$ Reversal: complexes cultured with iAC for $20 \mathrm{~h}$, and then cultured for a further $20 \mathrm{~h}$ without $\mathrm{A} A \mathrm{C}$. Total number of oocytes within each treatment ranged from 73 to 99 over five replications.

${ }^{b c}$ Within a column, values for treatments with different superscripts are significantly different $(P<0.05)$. The percentage of intermediates was not analysed.

Table 7. Development of bovine oocytes after suppressing meiotic resumption during collection procedures

\begin{tabular}{lccc}
\hline $\begin{array}{l}\text { Inhibitor added to } \\
\text { follicular fluid }\end{array}$ & $n$ & $\begin{array}{c}>2 \text { cell } \\
(\%)^{a}\end{array}$ & $\begin{array}{c}\text { Morulae and } \\
\text { blastocysts }(\%)^{b}\end{array}$ \\
\hline IBMX + iAC & 283 & 54 & 27 \\
None & 342 & 55 & 26
\end{tabular}

${ }^{a}$ The number of fertilized oocytes cleaving at least once $48 \mathrm{~h}$ after adding spermatozoa/total number of oocytes cultured $(n)$ averaged over three replicates.

"Number of morulae and blastocysts observed 6 days after fertilization/total oocytes $(n)$ cultured averaged over three replicates.

due to the time necessary for neutralization or clearance of invasive adenylate cyclase from the cells.

Developmental capacity of oocytes recovered from follicular fluid supplemented with IBMX + invasive adenylate cyclase

Experiment 5 was designed to evaluate possible detrimental effects of supplementing FF with IBMX plus invasive adenylate cyclase during the $2-3 \mathrm{~h}$ processing period on the subsequent developmental potential of oocytes. Treated oocytes were cultured in standard maturation medium for $24 \mathrm{~h}$, and were then fertilized and evaluated for development with a control group recovered from FF that was not supplemented. Oocytes in both groups cleaved and progressed to morula and blastocyst stages in vitro at equal frequencies (Table 7), indicating that supplementation of FF with IBMX plus invasive adenylate cyclase during collection was not detrimental.

\section{Phosphoprotein profiles of bovine oocytes}

In Expt 6, phosphoprotein profiles of oocytes maintained in meiotic arrest permanently by treatment with invasive adenylate cyclase or transiently by 8-Br-cAMP were analysed. Cumulus-enclosed oocytes were cultured for $9 \mathrm{~h}$ with either $20 \mathrm{U} \mathrm{iAC} \mathrm{ml}^{-1}, 10 \mathrm{mmol} 8$-Br-cAMP $\mathrm{l}^{-1}$ or in control medium. The treatment groups were transferred to similar

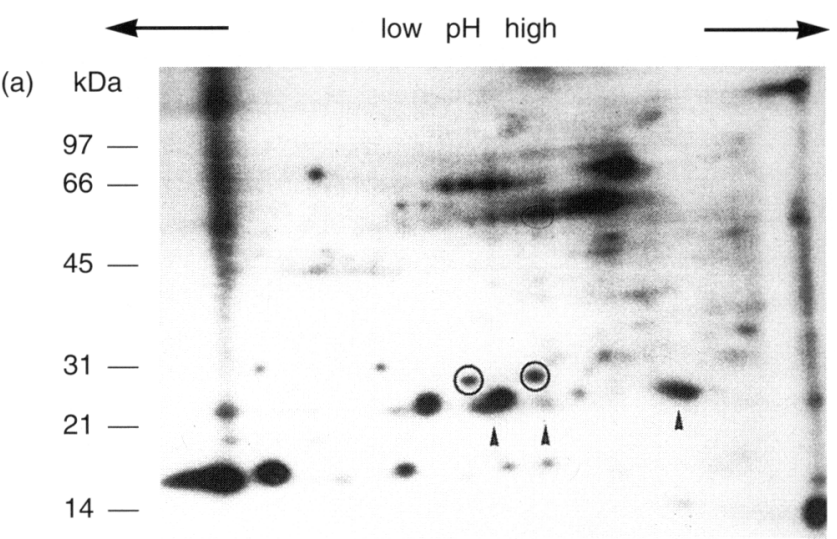

(b)

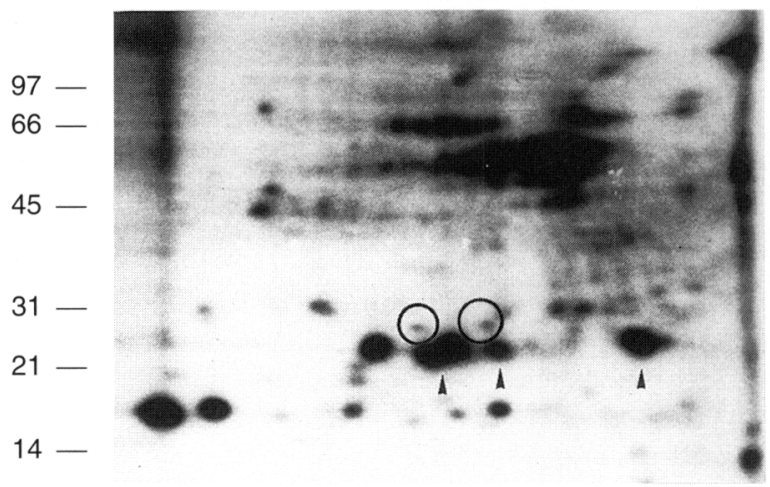

(c)

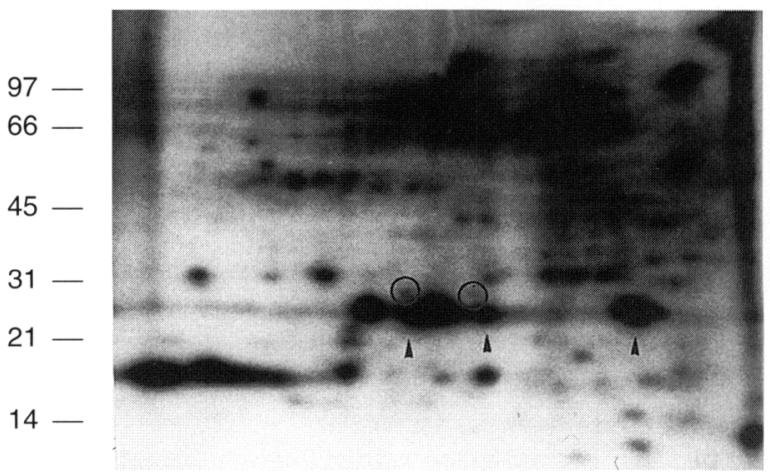

Fig. 1. Phosphoprotein profiles of bovine oocytes maintained in meiotic arrest with (a) (iAC) invasive adenylate cyclase, (b) transiently with 8-bromo-cAMP or (c) maturing spontaneously. Complexes were

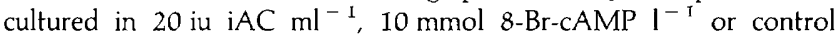
medium only for a total of $9 \mathrm{~h}$. For the last $2 \mathrm{~h} 15 \mathrm{~min}$, the complexes were transferred to phosphate-free medium supplemented with $\left[{ }^{32} \mathrm{P}\right]$ orthophosphate $\left(0.5 \mu \mathrm{Ci} \mathrm{ml}{ }^{-1}\right)$ and the appropriate inhibitor. At the end of culture, cumulus cells were removed and oocytes were washed in phosphate-free TL-Hepes several times. A gel is shown that used 75 oocytes per lane, selected from five replications, where each replicate used oocytes collected on separate days. Two $29 \mathrm{kDa}$ proteins (circled) are shown which become minimally phosphorylated in transiently arrested and spontaneously maturing oocytes. Arrows indicate 24,25 , and $2 \mathrm{kDa}$ proteins, which become more heavily phosphorylated in these two types of oocyte.

culture medium (phosphate-free TC-199 medium with added $\left[{ }^{32} \mathrm{P}\right]$ orthophosphate) for the last $2 \mathrm{~h} 15 \mathrm{~min}$ of culture. 
Table 8. Phosphoproteins in bovine oocytes maturing spontaneously, or maintained in germinal vesicle arrest with invasive adenylate cyclase (iAC), or transiently with 8-bromo-cAMP (8-Br-cAMP)

\begin{tabular}{lrcc}
\hline & \multicolumn{3}{c}{ Intensity $\left(\right.$ pixels $\left.\times 10^{3}\right) \pm \mathrm{SEM}^{\mathrm{a}}$} \\
\cline { 2 - 4 } $\begin{array}{l}\text { Protein } \\
\mathrm{kDa})\end{array}$ & Control & 8 -Br-cAMP & IAC \\
\hline 29 & $1 \pm 1$ & $5 \pm 0.2$ & $9 \pm 2$ \\
29 & $3 \pm 1$ & $4 \pm 2$ & $10 \pm 2$ \\
27 & $115 \pm 8$ & $107 \pm 10$ & $25 \pm 3$ \\
25 & $47 \pm 5$ & $43 \pm 6$ & $8 \pm 0.8$ \\
24 & $30 \pm 1$ & $29 \pm 6$ & $12 \pm 5$ \\
\hline
\end{tabular}

aixel intensities from gels were averaged over five replications, each consisting of gels run with oocytes recovered on different days.

Oocytes completely maintained in meiotic arrest (i.e., cultured in the presence of invasive adenylate cyclase, $90 \%$ at germinal vesicle stage at $9 \mathrm{~h}$ ) showed phosphoprotein profiles dissimilar to those of mature oocytes (Fig. 1; Table 8). These oocytes contained two prominent $29 \mathrm{kDa}$ phosphoproteins. Moreover, 27,25 and $24 \mathrm{kDa}$ proteins seen in mature oocytes were only minimally phosphorylated. Oocytes cultured with 8-Br-cAMP (maintained in meiotic arrest transiently) showed a phosphoprotein profile indistinguishable from control oocytes ( $10 \%$ at germinal vesicle stage), although they still had distinct germinal vesicles ( $85 \%$ intact germinal vesicles). Oocytes cultured with 8-Br-cAMP and those cultured in control medium showed maturation related phosphoproteins of 27,25 and $24 \mathrm{kDa}$ and two $29 \mathrm{kDa}$ proteins that were minimally phosphorylated compared with oocytes treated with invasive adenylate cyclase. These differences were obviously and consistently present in all five replications and indicated that, during transient delay of meiotic arrest, post-translational modifications in proteins related to maturation still occur.

\section{Discussion}

These studies have demonstrated that bovine oocytes can be reversibly maintained in meiotic arrest for an extended period by increasing $[\mathrm{CAMP}]_{i}$, therefore, cAMP may play a role in maintaining meiotic arrest in bovine oocytes as in other animals. Precautions to prevent premature commitment and choice of macromolecular media supplements were important aspects of this model. Use of a preculture treatment to prevent premature commitment to meiotic resumption did not impair the developmental potential of the oocytes. Bovine oocytes could not be kept in meiotic arrest with cAMP analogues such as 8-Br-cAMP or dibutyryl-cAMP (db-cAMP, data not shown); moreover, the phosphoprotein profiles of oocytes treated with 8-Br-cAMP clearly demonstrated that during transient arrest, post-translational modification of proteins, characteristic of maturing oocytes, continue to occur. These changes were not found in oocytes maintained in meiotic arrest with invasive adenylate cyclase.

Since the demonstration by Maller and Krebs (1977) that progesterone-induced oocyte maturation in Xenopus is inhibited by analogues of cAMP or phosphodiesterase inhibitors, numerous studies have been undertaken to elucidate possible functions of cAMP in maintaining mammalian oocytes in meiotic arrest (for review, see Schultz, 1988). It is now considered that intracellular concentrations of cAMP regulate the meiotic state of oocytes in many amphibians (Maller and Krebs, 1977) and mammals (Bornslaeger et al., 1986). Both progesterone-induced Xenopus oocyte maturation (Cicirelly and Smith, 1985) and spontaneous mouse oocyte maturation (Schultz et al., 1983) are accompanied by a decrease in intra-oocyte cAMP concentrations. Interference with this reduction prevents maturation (Schultz et al., 1983; Vivarelli et al., 1983).

Several studies (Rice and McGaughey, 1981; Homa, 1988; Sirard and First, 1988) carried out with oocytes of mammals with long oestrous cycles have failed to produce unequivocal results. Studies with oocytes (Torneil et al., 1984) and other cells in vitro (for review, see Lohmann and Walter, 1984) have shown that cAMP analogues may differ and that their effects on cell growth may be different from those of cellular cAMP. As reviewed by Lohman and Walter (1984), the action of cAMP and its analogues may be modified by the presence of serum, growth factors and hormones in culture medium, as we observed in the bovine model for studying meiotic regulation. The conflicting results reported on the effect of cAMP on oocyte maturation in domestic animals may result from different means of increasing $[c A M P]_{i}$ and different culture conditions (i.e., supplementation with serum or hormones). A further complication arises because bovine oocytes (Sirard and First, 1988), like those of other species (Dekel and Beers, 1980; Schultz et al., 1983), may commit to undergo meiosis during the collection process. These experiments were therefore conducted to describe the optimal culture conditions and means to increase $[\mathrm{CAMP}]_{i}$ for the study of effects of cAMP on bovine oocyte maturation.

The effects of increased $[\mathrm{cAMP}]_{\mathrm{i}}$ were investigated by using cumulus-intact bovine oocytes to compare different agents that increase $[c A M P]_{i}$ and determining how these substances interact with the macromolecular components of maturation medium. An attempt was also made to describe an optimal system for collecting these oocytes before culture. Homa (1988) demonstrated that 8-Br-cAMP was more potent than dibutyryl-cAMP in inhibiting bovine oocyte maturation. Preliminary studies showed that the same concentration of 8-Br-cAMP was more potent than db-cAMP when inhibition of progression beyond metaphase I was taken as an end point (data not shown). Our studies showed that 8-Br-cAMP, even when used at a concentration of $10 \mathrm{mmol} \mathrm{I}^{-1}$ (reported by Homa, 1988, to be the most effective concentration in maintaining bovine oocytes in meiotic arrest transiently) was a weak inhibitor of germinal vesicle breakdown of bovine oocytes, and invasive adenylate cyclase could inhibit germinal vesicle breakdown in bovine oocytes at a high frequency with reproducible results, when PVP and BSA were used as macromolecular supplements in culture medium. The correlation between CAMP concentration in oocytes and complexes and inhibition of germinal vesicle breakdown suggests that the enzyme acts through cAMP. By contrast, analogues of cAMP were less effective at preventing germinal vesicle breakdown. 
Fulka et al. (1993), by fusing mouse and bovine oocytes, showed that CAMP analogues fail to block germinal vesicle breakdown in both mouse and bovine oocyte nuclei within the same cytoplasm, even at doses as high as $5 \mathrm{mmol} \mathrm{l}^{-\mathrm{I}}$. However, both types of nuclei in the fused cell remained intact when cultured with IBMX ( $\left.1 \mathrm{mmol} \mathrm{1}^{-1}\right)$. These data suggest that, although bovine oocytes are insensitive to cAMP analogues and that maturation promoting factor created in bovine oocyte cytoplasm in the presence of these analogues causes germinal vesicle breakdown in both types of nucleus, bovine oocytes are sensitive to changes in concentration of cellular cAMP. The demonstration by Homa (1988) that IBMX, a phosphodiesterase inhibitor that causes accumulation of cAMP, was far more effective than either dibutyryl-cAMP or 8 -Br-cAMP in maintaining meiotic arrest is also in agreement with our results. The weak effect of 8 - $\mathrm{Br}$-cAMP may be due to its inability to cross bovine oocyte cell membranes. However, our measurements of the cAMP content of bovine oocytes showed that 8-Br-cAMP would accumulate in bovine oocytes very efficiently. Under the assay conditions used in the study reported here, similar concentrations of 8 -Br-cAMP and standard CAMP displaced ${ }^{125}$ I-labelled tracer to the same extent. The difference between the ability of 8-Br-cAMP and cAMP produced by invasive adenylate cyclase to maintain meiotic arrest may be due either to a difference in their subcellular distribution or in their ability to bind regulatory subunits on the cAMP-dependent protein kinase.

Earlier work with sheep (Moor and Heslop, 1981; Crosby et al., 1985; Moor, 1988) suggested that increased [CAMP $]_{i}$ would not inhibit oocyte maturation. Gonadotrophins included during the culture of ovine oocytes, rather than species difference, may account for the observed differences in patterns of $[\mathrm{cAMP}]_{\mathrm{i}}$. A positive stimulus from follicle cells induced by gonadotrophins may cause nuclear maturation without a concomitant fall in cAMP concentration (Downs et al., 1988) as we have observed in cattle.

An invasive adenylate cyclase has been used by Aberdam et al. (1987) at higher concentrations and in combination with IBMX and pertussis toxin on rat oocytes, and results comparable to those reported here were obtained. Aberdam et al. (1987) and others (Wheeler and Veldhuis, 1988; Gilboa-Ron et al., 1989; Gordon et al., 1989) showed that the invasive adenylate cyclase accumulated in the host cells, and was inactivated by the host cells in a time-dependent fashion if cells were transferred to a medium without invasive adenylate cyclase. The concentration of crude preparations of invasive adenylate cyclase used initially was inferred from a previous study using the same preparation (Wheeler and Veldhuis, 1988). This preparation and others were later characterized using maintenance of meiotic arrest in denuded bovine oocytes as a bioassay.

In rat cumulus-oocyte complexes, the inactivation and resulting recovery from the effect of invasive adenylate cyclase was detectable by $\mathrm{I} h$ and completed by $24 \mathrm{~h}$. When invasive adenylate cyclase plus IBMX were added to FF during collection and processing of the complexes, no long lasting effects were noted, since both the frequency of meiotic maturation and the formation of late stage preimplantation embryos were similar to control values. Thus, pretreatment during collection prevents spontaneous commitment of bovine oocytes to meiotic resumption and allows study of the mechanisms for meiotic regulation of the oocyte. Our demonstration that inhibition of oocyte maturation for $20 \mathrm{~h}$ in culture by invasive adenylate cyclase is reversible is in agreement with other findings that inhibition of oocyte maturation by either cAMP analogues or agents that increase $[c A M P]_{i}$ is reversible in mouse, rat, pig and hamster oocytes (Rice and McGaughey, 1981; Schultz et al., 1983; Aberdam et al., 1987; Racowsky, 1985b). Since reversal of the extended arrest was possible without compromising the developmental potential of most of the oocytes (Aktas et al., 1991b; Aktas, 1994), we consider that $[\mathrm{cAMP}]_{i}$ has a physiologically relevant role in maintaining meiotic arrest.

Meiotically arrested bovine oocytes have different phosphoprotein profiles from transiently arrested or spontaneously maturing oocytes. Differences in the phosphoproteins of mature and immature oocytes have been demonstrated in mouse (Bornslaeger et al., 1986) and frog (Maller and Smith, 1985) oocytes. That the oocytes incubated with 8-Br-cAMP show a phosphoprotein profile similar to that of control oocytes, even while possessing intact germinal vesicles, points to a possible reason why invasive adenylate cyclase, but not 8-Br-cAMP, maintains bovine oocytes in meiotic arrest for an extended period. Whether these phosphorylation and dephosphorylation events are the cause or the result of meiotic resumption is not yet clear. Similarly, it is not known whether two $29 \mathrm{kDa}$ phosphoproteins prominent in meiotically arrested oocytes are responsible for meiotic arrest or are the result of these events. Several proteins of $40-80 \mathrm{kDa}$, which were heavily phosphorylated in mature or maturing oocytes, are likely to be structural proteins (i.e. lamins) the phosphorylation of which is required for germinal vesicle breakdown to occur. The small gels used in this study do not facilitate further separation of these proteins.

In conclusion, the results of these studies suggest that $[\mathrm{cAMP}]_{\mathrm{i}}$ has a physiological role in maintaining meiotic arrest in bovine primary oocytes. They also present a useful model for further study of meiotic regulation in mammals with long oestrous cycles. Although previous work in domestic species has indicated that $[\mathrm{cAMP}]_{\mathrm{i}}$ plays a role in the maintenance of meiotic arrest, as in other animals, the work presented here provides a model for studying meiotic arrest when posttranslational mechanisms regulating meiotic resumption are held in abeyance. This is in strong contrast to the situation in which only a transient delay in meiotic resumption is induced.

The authors thank E. Hewlett for generously providing the adenylate cyclase and expert advice, P. Schoff and L. Hagemann for helpful comments on the manuscript, L. Hewitson for editorial assistance, J. Busby and B. Wagner for typing the manuscript and B. Haley for obtaining animal material. Research was supported by the College of Agricultural and Life Sciences, University of WisconsinMadison and CSRS-NRICGP Grant 88-37240-3740 to ML LeibfriedRutledge.

\section{References}

Aberdam E, Hanski E and Dekel N (1987) Maintenance of meiotic arrest in isolated rat oocytes by the invasive adenylate cyclase of Bordetella pertussis Biology of Reproduction 36 530-535 
Aktas, H (1994) Regulation of bovine oocyte maturation: maintenance of meiotic arrest and resumption of meiosis PhD Thesis, University of Wisconsin-Madison

Aktas H, Leibfried-Rutledge ML, Wheeler MB, Rosenkrans CF and First NL (1990) Maintenance of meiotic arrest in bovine oocytes Biology of Reproduction 41 (Supplement 1) Abstract 90

Aktas $\mathrm{H}_{i}$ Leibfried-Rutledge ML and First NL (1991a) Bovine oocyte maturation is regulated by interactions between cAMP, cumulus and granulosa Serono Symposium on Preimplantation Embryo Development Newton MA Abstract 53

Aktas H, Leibfried-Rutledge ML and Wheeler MB (1991b) Bovine oocytes arrested in meiosis in vitro retain developmental capacity Biology of Reproduction 41 (Supplement 1) Abstract 75

Bavister BD, Leibfried ML and Lieberman G (1983) Development of preimplantation embryos of golden hamsters in a defined culture medium Biology of Reproduction 28 235-247

Bornslaeger EA and Schultz RM (1985) Regulation of mouse oocyte maturation: effect of elevating cumulus cell cAMP on oocyte cAMP levels Biology of Reproduction 33 698-704

Bornslaeger EA, Mattei P and Schultz RM (1986) Involvement of cAMPdependent protein kinase and protein phosphorylation in regulation of mouse oocyte maturation Developmental Biology 114 453-462

Chen SC, Brown PR and Rosie DM (1977) Extraction procedures for use prior to HPLC nucleotide analysis using microparticle chemically bonded packing Journal of Chromatography 15 218-221

Cho WK, Stern S and Biggers JD (1974) Inhibitory effect of dibutyryl cAMP on mouse oocyte maturation in vitro Joumal of Experimental Zoology 187 383-386

Cicirelly MF and Smith LD (1985) Cyclic AMP levels during the maturation of Xenopus oocytes Developmental Biology 108 254-258

Crosby IM, Moor RM, Heslop JP and Osborn JC (1985) cAMP in ovine oocytes: localization of synthesis and its action on protein synthesis, phosphorylation, and meiosis Journal of Experimental Zoology 234 307-318

Dekel N and Beers WH (1980) Development of the rat oocyte in vitro: inhibition and induction of maturation in the presence or absence of the cumulus oophorus Developmental Biology 75 247-254

Downs SM, Daniel SAJ and Eppig JJ (1988) Induction of maturation in cumulus cell-enclosed mouse oocytes by follicle-stimulating hormone and epidermal growth factor: evidence for a positive stimulus of somatic cell origin Journal of Experimental Zoology 245 86-96

Eyestone WH and First NL (1989) Co-culture of early cattle embryos to the blastocyst stage with oviductal tissue or in conditioned medium fournal of Reproduction and Fertility $85715-720$

Fulka J Jr, Leibfried-Rutledge ML and First NL (1993) Control of germinal vesicle breakdown in bovine $\times$ murine hybrid oocytes Reproduction. Nutrition and Development 33 411-417

Gilboa-Ron A, Rogel A and Honsla E (1989) Bordetella pertussis adenylate cyclase inactivation by the host cell Biochemical Journal 262 25-31

Gordon WM, Young WW, Lechler SM and Gray MC (1989) Adenylate cyclase toxins from Bacillus anthracis and Bordetella pertussis Journal of Biological Chemistry $26414792-14796$

Homa ST (1988) Effects of cyclic AMP on the spontaneous meiotic maturation of cumulus-free bovine oocytes cultured in chemically defined medium Journal of Experimental Zoology 248 222-23I

Kuyt JRM, Kruip TAM and de Jong-Brink M (1988) Cytochemical localization of adenylate cyclase in bovine cumulus-oocyte complexes Experimental Cell Research 174 139-145

Laemmli UK (1970) Cleavage of structural proteins during assembly of head of bacteriophage T4 Nature 227 680-685

Leibfried ML and Bavister BD (1982) The effect of epinephrine and hypotaurine on in vitro fertilization in the hamster Journal of Reproduction and Fertility 66 $87-93$
Leibfried ML and First NL (1979) Characterization of bovine follicular oocytes and their ability to mature in vitro Journal of Animal Science 48 76-86

Lenz RW, Ball GD, Leibfried ML, Ax RL and First NL (1983) In vitro maturation and fertilization of bovine oocytes are temperature-dependent processes Biology of Reproduction 29 173-179

Lohmann SM and Walter UW (1984) Regulation of the cellular and subcellular concentrations and distribution of cyclic nucleotide-dependent protein kinase. In Advances in Cyclic Nucleotide and Protein Phosphorylation Research Vol. 18 pp 63-115 Ed. P Greengard. Raven Press, New York

Maller JL and Krebs EJ (1977) Progesterone-stimulated meiotic cell division in Xenopus oocytes Journal of Biological Chemistry 252 1712-1718

Maller JL and Smith DS (1985) Two-dimensional polyacrylamide gel analysis of changes in protein phosphorylation during maturation of Xenopus oocytes Developmental Biology 109 150-156

Moor RM (1988) Regulation of the meiotic cycle in oocytes of domestic mammals. Annals of the New York Academy of Science 541 248-258

Moor RM and Heslop JP (1981) Cyclic AMP in mammalian follicle cells and oocytes during maturation Journal of Experimental Zoology 216 205-208

Parrish JJ, Susko-Parrish JL, Leibfried-Rutledge ML, Critser ES, Eyestone WH and First NL (1986) Bovine in vitro fertilization with frozen-thawed semen Theriogenology 25 591-600

Racowsky C (1984) Effect of forskolin on the spontaneous maturation and cyclic AMP content of rat oocyte-cumulus complexes Journal of Reproduction and Fertility $\mathbf{7 2}$ 107-116

Racowsky C (1985a) Effect of forskolin on maintenance of meiotic arrest and stimulation of cumulus expansion, progesterone and cyclic AMP production by pig oocyte-cumulus complexes Journal of Reproduction and Fertility $\mathbf{7 4}$ 9-21

Racowsky C (1985b) Effect of forskolin on the spontaneous maturation and cyclic AMP content of hamster cocyte-cumulus complexes Journal of Experimental Zoology 234 87-96

Rice C and McGaughey RW (1981) Effect of testosterone and dibutyryl cAMP on the spontaneous maturation of pig oocytes Journal of Reproduction and Fertility 62 245-256

Schoff PK, Cheetham J and Lardy HA (1989) Adenylate kinase activity in ejaculated bovine sperm flagella Journal of Biological Chemistry 264 60866091

Schultz RM (1988) Role of protein phosphorylation in meiotic maturation of mouse oocytes in vitro Annals New York Academy of Sciences 541 217-227

Schultz RM, Montgomery RR and Belanoff JR (1983) Regulation of mouse oocyte meiotic maturation: implication of a decrease in oocyte CAMP and protein dephosphorylation in commitment to resume meiosis Developmental Biology 97 264-273

Sirard MA (1990) Temporary inhibition of meiosis resumption in vitro by adenylate cyclase stimulation in immature bovine oocytes Theriogenology 33 757-767

Sirard MA and First NL (1988) In vitro inhibition of oocyte nuclear maturation in the bovine Biology of Reproduction 39 228-234

Sirard MA, Parrish JJ, Ware CB, Leibfried-Rutledge ML and First NL (1988) The culture of bovine oocytes to obtain developmentally competent embryos Biology of Reproduction 39 546-552

Tornell J, Brannstrom M and Hillensjo T (1984) Different effects of cyclic nucleotide derivatives upon the rat oocyte-cumulus complex in vitro Acto Physiologica Scandinavica 122 507-513

Vivarelli E, Conti M, DeFelici M and Siracusa G (1983) Meiotic resumption and intracellular cAMP levels in mouse oocytes treated with compounds which act on CAMP metabolism Cell Differentiation 12 271-276

Wheeler MB and Veldhuis JD (1988) Interactions of protein kinase C with receptor and non-receptor-mediated cyclic AMP generation in swine granulosa cells Molecular Cell Endocrinology 58 195-203 